\title{
The Delta Scuti Network: Steps Towards Successful Asteroseismology of Delta Scuti Stars
}

W. Zima, M. Breger, K. Bischof, F. Rodler, A. Stankov, A. A. Pamyatnykh, V. Antoci, D. Lorenz, M. Sperl, R. Zechner

Institut für Astronomie, Türkenschanzstr. 17, A-1180 Wien, Austria, zima@astro.univie.ac.at

R. Garrido

Instituto de Astrofisica de Andalucia, CSIC, Apdo. 3004, E-18080 Granada, Spain

M. Wood

Dept. of Physics and Space Science, Florida Institute of Technology, Melbourne, FL 32901, U.S.A.

G. Handler

SAAO, P.O. Box 9, Observatory 7935, Cape Town, South Africa

R.R. Shobbrook

School of Physics, University of Sydney 2006, Australia

\begin{abstract}
The Delta Scuti Network (DSN) is a collaboration of astronomers all around the globe who study and observe short-period variables. The field of scientific research includes high-precision photometric and spectroscopic global campaigns, mode identification techniques, and asteroseismological pulsation modeling. We present results for three stars that are receiving the most attention at the moment: $4 \mathrm{CVn}, \mathrm{BI} \mathrm{CMi}$, and 44 Tau. Our results demonstrate that a large number of simultaneously excited nonradial oscillations in stars on and above the main sequence can be detected by conventional means.
\end{abstract}

\section{4 CVn (HD 107904, V $=6^{m} .0$, F3 III-IV)}

For $4 \mathrm{CVn}$, which was observed by the DSN during 1996 (Breger et al., 1999) and 1997 (Breger \& Hiesberger, 1999) we found a total of 34 frequencies, of which 16 are linear combinations. This star is highly evolved and hundreds of low-degree oscillation modes are predicted by theory to be excited. The fact that only a fraction of this number seems to be excited raises the problem of a mode selection mechanism.

This star's unusually strong amplitude variability has been monitored since 1966 (Breger, 2000). The amplitudes vary on timescales of several years with an annual change up to 12 per cent and a change of 40 per cent in a decade. The reason for the amplitude variability is at present unknown. 
For 10 oscillation modes, the pulsational degree, $\ell$, was derived from the phase differences in the Strömgren filters $v$ and $y$ (Garrido et al., 1990). We also detected a high number of combination frequencies $f_{i} \pm f_{j}$, which lie outside the main pulsation range. There may be a connection between the strong amplitude variability and the combination frequencies through a transfer of energy between the modes.

\section{BI CMi (HD 66853, $\left.\mathrm{V}=9^{m} .2, \mathrm{~F} 2\right)$}

Two global campaigns on BI CMi in 1998/99 and 1999/2000, covering a total of more than 1000 hours, have resulted in the detection of 29 oscillation frequencies. This star was chosen on account of its position close to the cool border of the instability strip. Would this star exhibit Delta Scuti (p-mode) and Gamma Doradus (g-mode) type pulsations simultaneously?

The majority of statistically significant frequencies was found to be in a range of 4.7 to $13.1 \mathrm{c} \mathrm{d}^{-1}$ (55 to $150 \mu \mathrm{Hz}$ ). In addition, a number of combination frequencies were detected. Also, a very low frequency with a value of $1.66 \mathrm{~cd}^{-1}(19.2 \mu \mathrm{Hz})$ was found, which is typical for Gamma Doradus stars. Since the value of this frequency cannot be identified with a linear combination of other frequencies, g-mode pulsation is suspected, but rotational modulation of abundance spots cannot be ruled out.

\section{44 Tau (HD 26322, V $=5^{m} .4$, F2 IV-V)}

44 Tau was observed photometrically by the DSN in 2000/01 during more than 200 hours from the following observatories: the Vienna Automatic Photoelectric Telescope located in Arizona, the Sierra Nevada Observatory and the Mt. Ege Observatory, Turkey.

This star shows an extremely low value of $v \sin i=5 \mathrm{~km} \mathrm{~s}^{-1}$ (Smith 1979), indicating either an extremely low value of the inclination angle $i$ or intrinsically slow rotation. In the latter case, mode identification would be simplified, because we would see groups of equally spaced rotationally split modes in the frequency spectrum, without overlapping of adjacent radial orders.

A preliminary analysis of the new 44 Tau data enabled us to detect 17 frequencies, of which 6 are linear combinations. The combination frequencies, $f_{i}-f_{j}$, could not be detected due to higher observational noise in the lowfrequency region.

\section{References}

Breger, M. 2000, MNRAS, 313, 129

Breger, M. \& Hiesberger, F. 1999, A\&AS, 135, 546

Breger, M., et al. 1999, A\&A, 349, 225

Garrido, R., et al. 1990, A\&A, 234, 262

Smith, M.A. 1979, ApJ, 254, 242 